\title{
Pseudomonas infections among hospitalized adults in Latin America: a systematic review and meta-analysis
}

\author{
Alfredo Ponce de Leon ${ }^{1}$, Sanjay Merchant ${ }^{2}$, Gowri Ramann ${ }^{3,4}$, Esther Avendano ${ }^{3 *}$, Jeffrey Chan ${ }^{3}$, \\ Griselda Tepichin Hernandez ${ }^{2}$ and Eric Sarpong ${ }^{2}$
}

\begin{abstract}
Background: Treatment of resistant Pseudomonas aeruginosa infection continues to be a challenge in Latin American countries (LATAM). We synthesize the literature on the use of appropriate initial antibiotic therapy (AIAT) and inappropriate initial antibiotic therapy (IIAT) in $P$. aeruginosa infections, and the literature on risk factors for acquisition of resistant $P$. aeruginosa among hospitalized adult patients in LATAM.

Methods: MEDLINE, EMBASE, Cochrane, and LILAC were searched between 2000 and August 2019. Abstracts and full-text articles were screened in duplicate. Random effects meta-analysis was conducted when studies were sufficiently similar.

Results: The screening of 165 citations identified through literature search yielded 98 full-text articles that were retrieved and assessed for eligibility, and 19 articles conducted in Brazil (14 articles), Colombia (4 articles), and Cuba (1 article) met the inclusion criteria. Of 19 eligible articles, six articles (840 subjects) examined AIAT compared to IIAT in $P$. aeruginosa infections; 17 articles (3203 total subjects) examined risk factors for acquisition of resistant $P$. aeruginosa; and four articles evaluated both. Four of 19 articles were rated low risk of bias and the remaining were deemed unclear or high risk of bias. In meta-analysis, AIAT was associated with lower mortality for P. aeruginosa infections (unadjusted summary OR $0.48,95 \% \mathrm{Cl} 0.28-0.81 ; I^{2}=59 \%$ ), compared to IIAT and the association with mortality persisted in subgroup meta-analysis by low risk of bias ( 3 articles; unadjusted summary $\mathrm{OR} 0.46,95 \% \mathrm{Cl} 0.28-0.81 ;\left.\right|^{2}=0 \%$ ). No meta-analysis was performed for studies evaluating risk factors for acquisition of resistant $P$. aeruginosa as they were not sufficiently similar. Significant risk factors for acquisition of resistant $P$. aeruginosa included: prior use of antibiotics (11 articles), stay in the intensive care unit (ICU) (3 articles), and comorbidity score (3 articles). Outcomes were graded to be of low strength of evidence owing to unclear or high risk of bias and imprecise estimates.
\end{abstract}

Conclusion: Our study highlights the association of AIAT with lower mortality and prior use of antibiotics significantly predicts acquiring resistant $P$. aeruginosa infections. This review reinforces the need for rigorous and structured antimicrobial stewardship programs in the LATAM region.

Keywords: Pseudomonas aeruginosa, Appropriate, Inappropriate, Latin America, Risk factors, Antibiotic therapy, Resistance

\footnotetext{
* Correspondence: eavendano@tuftsmedicalcenter.org

${ }^{3}$ Institute for Clinical Research and Health Policy Studies, Center for Clinical

Evidence Synthesis, Tufts Medical Center, 800 Washington St, Boston, MA

02111, USA

Full list of author information is available at the end of the article
}

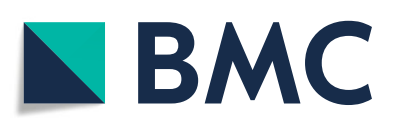

(c) The Author(s). 2020 Open Access This article is licensed under a Creative Commons Attribution 4.0 International License, which permits use, sharing, adaptation, distribution and reproduction in any medium or format, as long as you give appropriate credit to the original author(s) and the source, provide a link to the Creative Commons licence, and indicate if changes were made. The images or other third party material in this article are included in the article's Creative Commons licence, unless indicated otherwise in a credit line to the material. If material is not included in the article's Creative Commons licence and your intended use is not permitted by statutory regulation or exceeds the permitted use, you will need to obtain permission directly from the copyright holder. To view a copy of this licence, visit http://creativecommons.org/licenses/by/4.0/ The Creative Commons Public Domain Dedication waiver (http://creativecommons.org/publicdomain/zero/1.0/) applies to the data made available in this article, unless otherwise stated in a credit line to the data. 


\section{Background}

Antimicrobial resistant Gram-negative infections continue to increase and pose a major public health problem in LATAM $[1,2]$. Analysis of 245 P. aeruginosa isolates from a 2018 Program to Assess Ceftolozane/Tazobactam Susceptibility (PACTS) database on 6 countries (Argentina, Brazil, Chile, Costa Rica, Mexico, and Panama) highlight the relatively high rates of antimicrobial resistance in Latin America (Table 1) [3, 4]. Among Gram-negative pathogens, resistant $P$. aeruginosa is the most common cause of nosocomial and healthcare associated infections (HAIs) [2]. Resistant $P$. aeruginosa is associated with increased mortality and significant costs [3]. The treatment of resistant $P$. aeruginosa, an opportunistic pathogen with an ability to rapidly develop resistance to multiple classes of antibiotics, is especially challenging.

IIAT in pneumonia, sepsis, and other infections can adversely impact health outcomes, increase length of stay in the hospital, and can result in increased mortality [5]. Identifying factors predicting the risk for acquisition of resistant $P$. aeruginosa or identifying subgroups of patients who are at an increased risk for such acquisition can help facilitate surveillance and optimize therapeutic management. Additionally, the long-term effectiveness of antimicrobials is reliant on appropriate and controlled use, and effective antibiotic stewardship, as stipulated in current guidelines [6]. There is a considerable gap in knowledge regarding the role of IIAT use in the LATAM region as well as risk factors associated with occurrence of resistant $P$. aeruginosa in hospitalized patients in the LATAM region.

Although available data suggest that AIAT reduce mortality and improve outcomes, to our knowledge, no study has systematically synthesized their specific contribution in LATAM. A number of individual studies exist in the public domain demonstrating consequences of AIAT versus IIAT as well as those that examine risk factors associated with resistant $P$. aeruginosa infections in LATAM. However, there has not been a comprehensive and systematic evaluation of the contemporary literature on this topic among hospitalized adult patients in LATAM.

This systematic literature review focuses on LATAM countries and critically examines two objectives: 1) the role of AIAT, as compared with IIAT in hospitalized adult patients undergoing initial treatment for nosocomial or hospital-acquired or healthcare-associated $P$. aeruginosa infections; 2) examine available evidence on risk factors associated with acquisition of resistant $P$. aeruginosa infection among hospitalized adult patients.

Table 1 Susceptibility of P. aeruginosa (245 isolates) Pathogens to Antimicrobials in the Latin America region ${ }^{\mathrm{a}}$ from the 2018 PACTS Database

\begin{tabular}{|c|c|c|c|c|c|c|c|c|c|}
\hline \multirow[b]{3}{*}{ Antimicrobial agent } & \multirow{2}{*}{\multicolumn{2}{|c|}{$\mathrm{MIC}(\mathrm{mg} / \mathrm{mL})$}} & \multirow{3}{*}{ Range } & \multicolumn{6}{|c|}{ \% Susceptible } \\
\hline & & & & \multicolumn{3}{|c|}{$\overline{C L S I}{ }^{c}$} & \multicolumn{3}{|c|}{ EUCAST $^{c}$} \\
\hline & $50 \%$ & $90 \%$ & & $\% \mathrm{~S}$ & $\% \mathrm{l}$ & $\% R$ & $\% \mathrm{~S}$ & $\% \mathbf{l}$ & $\% R$ \\
\hline Ceftolozane-tazobactam & 0.5 & 4 & 0.12 to $>32$ & 90.2 & 0.8 & 9.0 & 90.2 & & 9.8 \\
\hline Amikacin & 4 & $>32$ & 0.5 to $>32$ & 83.6 & 1.2 & 15.2 & 79.1 & 4.5 & 16.4 \\
\hline Ampicillin-sulbactam & $>64$ & $>64$ & 8 to $>64$ & & & & & & \\
\hline Aztreonam & 8 & $>16$ & 0.25 to $>16$ & 64.1 & 13.5 & 22.4 & 77.6 & & 22.4 \\
\hline Cefepime & 2 & 32 & 0.25 to $>256$ & 80.0 & 7.8 & 12.2 & 80.0 & & 20.0 \\
\hline Ceftazidime & 2 & $>32$ & 0.5 to $>32$ & 77.1 & 6.1 & 16.7 & 77.1 & & 22.9 \\
\hline Ceftriaxone & $>8$ & $>8$ & 1 to $>8$ & & & & & & \\
\hline Ciprofloxacin & 0.12 & $>16$ & $\leq 0.03$ to $>16$ & 68.9 & 4.5 & 26.6 & 68.9 & & 31.1 \\
\hline Colistin & 0.5 & 1 & $\leq 0.06$ to 2 & 100.0 & & 0.0 & 100.0 & & 0.0 \\
\hline Doripenem & 0.5 & 8 & $\leq 0.06$ to $>8$ & 78.4 & 7.3 & 14.3 & 72.2 & 6.1 & 21.6 \\
\hline Gentamicin & 2 & $>16$ & $\leq 0.12$ to $>16$ & 77.0 & 2.0 & 20.9 & 77.0 & & 23.0 \\
\hline Imipenem & 1 & $>8$ & $\leq 0.12$ to $>8$ & 74.3 & 3.7 & 22.0 & 78.0 & & 22.0 \\
\hline Levofloxacin & 0.5 & 32 & 0.03 to $>32$ & 64.3 & 5.7 & 29.9 & 64.3 & & 35.7 \\
\hline Meropenem & 0.5 & 16 & $\leq 0.015$ to $>32$ & 73.5 & 7.8 & 18.8 & 73.5 & 12.2 & 14.3 \\
\hline Piperacillin-tazobactam & 4 & 128 & 0.25 to $>128$ & 74.3 & 12.2 & 13.5 & 74.3 & & 25.7 \\
\hline Tigecycline & 8 & $>8$ & 1 to $>8$ & & & & & & \\
\hline
\end{tabular}

${ }^{a}$ Includes only Latin American countries (i.e., Argentina, Brazil, Chile, Costa Rica, Mexico, and Panama) with available data in the PACTS database

b PACTS Program to Assess Ceftolozane/Tazobactam Susceptibility

c Criteria as published by CLSI [2019] and EUCAST [2019]

CLSI Clinical and Laboratory Standards Institute; EUCAST European Committee on Antimicrobial Susceptibility Testing; MIC minimum inhibitory concentration;

PACTS Program to Assess Ceftolozane/Tazobactam Susceptibility 


\section{Methods}

We followed standard systematic review methods of conduct and reporting as detailed in the Preferred Reporting Items for Systematic Reviews and MetaAnalyses (PRISMA) criteria [7]. A priori protocol was created and retained for internal reference.

\section{Search strategy}

We performed a comprehensive systematic literature search in the MEDLINE ${ }^{\oplus}$ Cochrane Central, and EMBASE $^{\oplus}$ databases for citations indexed from January 2000 through August 2019 without any language restriction. Additional searches were conducted in the Literatura Latino Americana em Ciências da Saúde (LILAC) and Biblioteca Regional de Medicina (BIREME) databases for citations indexed from January 2000 to August 2019 to identify Spanish and other foreign language articles from the LATAM region. We searched for contemporary articles in this area beginning from 2000 because a previous study that evaluated global publications on this topic identified articles published from mid-to-late 2000 onwards [8]. To ensure completeness of our systematic literature search, we reviewed reference lists of eligible studies and eligible systematic reviews identified from the aforementioned sources. We sought input from an infectious disease clinical expert in the LATAM region with regard to any potentially eligible publications. When eligible, we also considered abstracts from conference proceedings. Separate searches were conducted for each of the objectives. Supplemental Table 1 lists the search strategy terms related to the pathogen ( $P$. aeruginosa), site of infection (urinary tract, intra-abdominal, bloodstream, and pneumonia), initial therapy (inappropriate, appropriate, adequate, or effective), and antibacterial drug therapy. Supplemental Table 2 lists terms related to $P$. aeruginosa, mode of infection (nosocomial, hospital-acquired, healthcare-acquired, hospitalassociated, healthcare-associated), and risk factor assessment (risk factors, predict, risk score, risk assessment, and multivariate analysis) and was limited to the LATAM region (Latin America and Caribbean countries). Articles published in Spanish and Portuguese languages, the most common languages from the LATAM region were translated by one of the authors (EEA).

\section{Study selection}

We screened all citations in duplicate. During initial rounds of citation screening, we implemented a training session where all researchers screened the same set of articles and iteratively continued training until all researchers agreed with the nuances of citation screening and selection. Disagreements were resolved in group meetings in discussion with a senior reviewer.

\section{Study eligibility criteria}

We included articles evaluating adults $\geq 18$ years of age hospitalized in a ward, intensive or critical care unit with confirmed acquisition of nosocomial or hospitalacquired or healthcare-associated $P$. aeruginosa infection. For studies with multiple publications, we included those with the longest follow-up, largest sample size, or both. Eligible data from observational (prospective and retrospective studies) and trials were included.

We excluded studies that were conducted among pediatric population. International studies that did not report stratified data by LATAM region were excluded. Non-human studies, narrative reviews, cross-sectional studies, case reports, editorials, letters and notes/comments were also excluded. In addition to the above common eligibility criteria, we specified the following additional criteria according to each of the objectives:

\section{Study eligibility criteria for AIAT vs. IIAT}

Studies of adult patients with susceptible, resistant, or MDR $P$. aeruginosa infections of the following sites: respiratory, intra-abdominal, bloodstream, and urinary tract were included. Studies that compared patients who received AIAT and IIAT and reported outcomes of interest were included. The outcomes of interest included: mortality (primary outcome), clinical success, microbiologic eradication, length of stay (hospital or ICU), and cost.

Studies with patients that may have been exposed to initial antimicrobial therapy before being hospitalized were excluded.

\section{Study eligibility criteria for risk factors for acquiring resistant $P$. aeruginosa}

Articles reporting risk factors that predicted acquisition of resistant $P$. aeruginosa, with or without extended-spectrum beta-lactamases (ESBLs) were included. Resistant $P$. aeruginosa infection was defined as cephalosporin-resistant and/or piperacillin/tazobactam-resistant and/or carbapenem-resistant, and/or multi-drug (MDR) or and/or extremely drug-resistant (XDR) $P$. aeruginosa infection. Studies reporting any of the following comparisons were included: MDR/XDR versus resistant $P$. aeruginosa, $\mathrm{MDR} / \mathrm{XDR} /$ resistant versus susceptible $P$. aeruginosa, and MDR/XDR/resistant versus any control.

\section{Data collection}

All articles identified as possibly meeting the eligibility criteria were then extracted independently by one experienced reviewer and the data were then validated by a second reviewer.

Pertinent data including study design, country where study was conducted, funding source, participant characteristics, identified pathogen, source of infection, site of infection, inclusion criteria, exclusion criteria, co- 
occurring conditions, co-morbidity scores, and unadjusted or adjusted analysis were extracted into customized forms in Excel $^{\circ}$. Data extraction for AIAT versus IIAT included author's definition of AIAT or IIAT (individual study definitions of AIAT and IIAT were accepted), percentage of patients receiving AIAT or IIAT. We tested the data extraction forms on several studies and revised as necessary before full data extraction. Any missing information was deemed as not reported information. Any disagreements were resolved by discussion amongst the team to achieve consensus.

\section{Assessment of study quality}

We assessed the methodological quality of each study based on predefined criteria. We used the Agency for Healthcare Research and Quality (AHRQ) risk of bias tool, which asks about risk of selection bias, performance bias, detection bias, attrition bias, reporting bias, and other potential biases [9]. Risk of bias was assessed by two reviewers and disagreements were resolved in group meetings in discussion with a senior reviewer.

\section{Assessment of overall strength of evidence}

The Grading of Recommendations Assessment, Development and Evaluation (GRADE; https://www.gradeworkinggroup.org/) was employed to assess the strength of overall evidence for outcomes that were assessed in meta-analysis and graded into one of the following: High, Moderate, Low or Very Low. The GRADE assessments were conducted in duplicate and disagreements were resolved in consensus.

\section{Data analysis}

When eligible studies were clinically heterogeneous in terms of outcomes or comparators, data were presented descriptively using systematic review instead of metaanalysis. We conducted meta-analyses of eligible studies that assessed sufficiently similar populations and outcomes and presented results of random effects metaanalysis as this model can estimate the mean of a distribution of true effect size (ES) [10]. For unadjusted results evaluating AIAT versus IIAT, we estimated odds ratio (OR) and its 95\% confidence interval (CI) from data reported in individual studies. If available, we combined adjusted OR across studies. We tested betweenstudy statistical heterogeneity with the Q statistic $(p<$ 0.10 was deemed statistically significant) and quantified its extent with $\mathrm{I}^{2}$ and 95\% CI [11]. We conducted analyses in Stata version 14 (StataCorp, College Station, Texas) with the metan, metareg, and metabias functions. For risk factors for acquiring resistant $P$. aeruginosa, we evaluated results reported in multivariable analyses that adjusted for potential confounders.

\section{Results}

Baseline study and population characteristics

The literature search identified a total of 165 citations; of which, 98 full-text articles were retrieved and assessed for eligibility. The systematic review included 19 articles (6 articles for AIAT versus IIAT [12-17]; 17 articles for acquisition of resistant or multidrug-resistant $P$. aeruginosa $[12,14-16,18-30]$ and 4 articles reported both outcomes [12, 14-16]) that met final eligibility criteria (Fig. 1).

\section{AIAT vs. IIAT}

Seven eligible studies (published in six articles) included 885 hospitalized adults with $P$. aeruginosa infections. Included studies were conducted between 2005 and 2012 in Brazil (5 studies [12, 14, $15,17,31]$ ) and Colombia (1 study). One study was retrospective cohort and five studies were casecontrol designs (Table 2). The underlying site of infection was bloodstream infection (3 studies [12, 15, $31]$ ), respiratory (2 studies [13, 17]) and information was not reported in one study [14]. The average age of patients among included studies ranged between 50 and 73 years.

Included studies heterogeneously defined appropriate use of antibiotic therapy. The common definition was use of initial antimicrobial agent within a specific number of hours (between 24 and $<48 \mathrm{~h}$ ) after index blood culture and/or that initial antibiotic displayed susceptibility against pathogen of interest in subsequent in vitro examination (Table 2 ). All studies reported 30-day mortality data and no studies reported data on treatment response, cost, or length of stay outcomes. The risk of bias was, generally, high or unclear. For example, most of these studies failed to clearly report the methods employed (Supplemental Table 3).

\section{Mortality outcomes in AIAT vs. IIAT Unadjusted data}

The meta-analysis of unadjusted data found that use of AIAT for $P$. aeruginosa infections, as compared with IIAT significantly decreased mortality, but with heterogeneity (6 studies; summary OR $0.48,95 \%$ CI $0.28-0.81 ; \mathrm{I}^{2}=58.6,95 \%$ CI $0-83 \%$ ) (Fig. 2). Only one study reported the outcome of in-hospital mortality [13]. The subgroup analyses identified that AIAT significantly decreased 30-day mortality, but with heterogeneity, when initiation of antibiotics was within $48 \mathrm{~h}$ of index blood culture (3 articles [12, 13, 16]; summary OR $0.46,95 \%$ CI $0.32-0.67 ; \mathrm{I}^{2}=0.0,95 \%$ CI $0-85 \%$ ), in blood stream infections (3 articles [12, 15, 16]; summary OR 0.36 , 95\% CI $0.19-0.68 ; \mathrm{I}^{2}=43.7,95 \%$ CI $\left.0-81 \%\right)$, and 


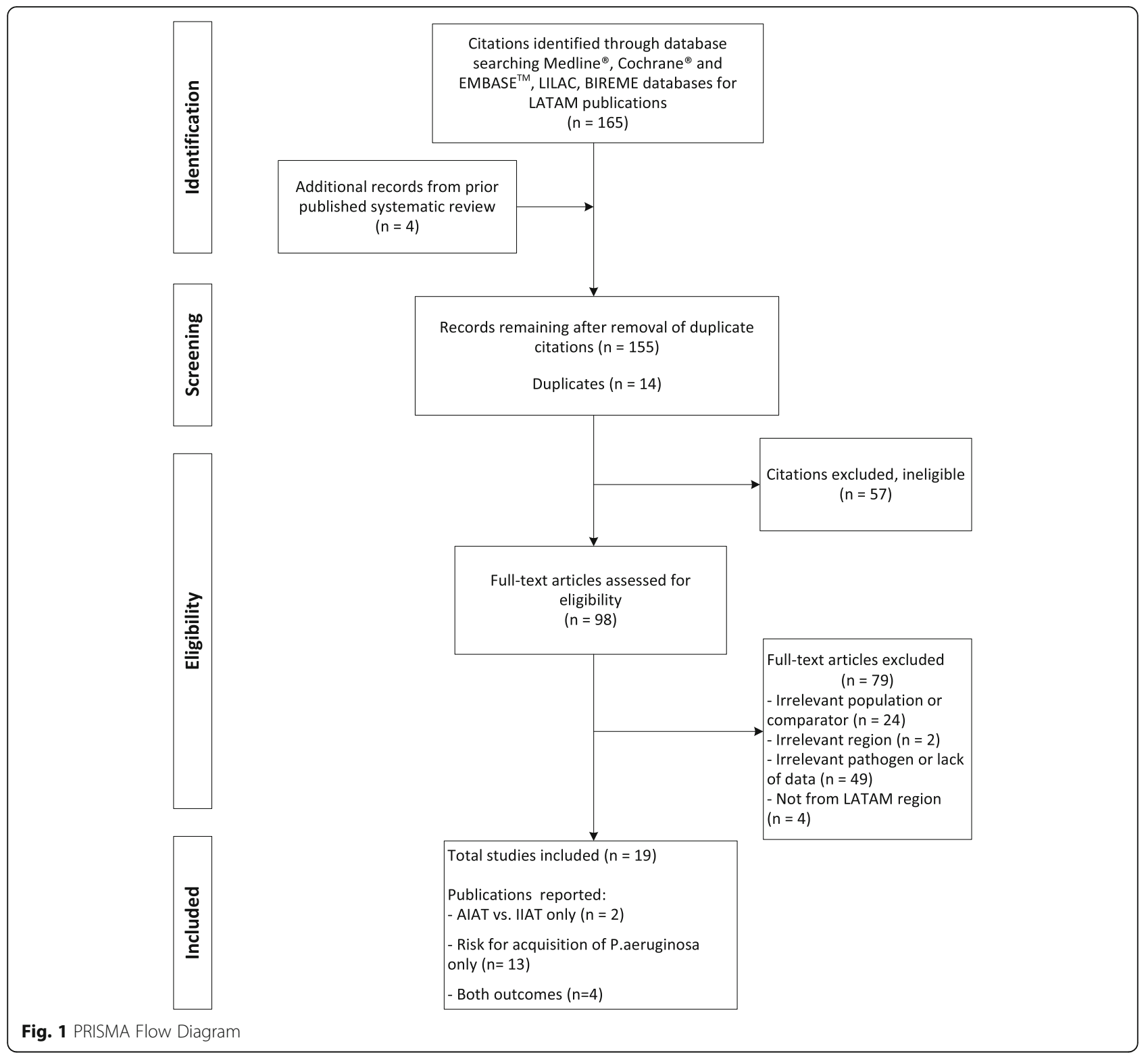

among patients admitted to the hospital wards (3 articles $[12,15,16]$; summary OR $0.36,95 \%$ CI $0.19-0.68 ; \mathrm{I}^{2}=58.6,95 \%$ CI $\left.0-81 \%\right)$. In subgroup meta-analysis by low risk of bias, AIAT was associated with lower mortality for $P$. aeruginosa infections ( 3 articles; unadjusted summary OR 0.46, 95\% CI $0.28-0.81 ; \mathrm{I}^{2}=89.7,95 \%$ CI $0-85 \%$ ), while metaanalysis of unclear risk of bias showed AIAT was not associated with lower mortality for $P$. aeruginosa infections ( 2 articles; unadjusted summary OR 0.45 , 95\% CI $0.08-2.62$; $\mathrm{I}^{2}=89.7$, 95\% CI NA) compared to IIAT. Meta-regression found no relationship between age (in years) or percent of patients with ICU admissions and a decrease in mortality with AIAT.

\section{Adjusted data}

Two studies reported an adjusted association between IIAT and mortality in P. aeruginosa infections and found a significantly increased mortality with IIAT, as compared with AIAT (Adjusted hazard ratio [HR] 2.95, 95\% CI 1.63-5.33 [13]; adjusted HR 5.54, 95\% CI 2.15-14.56 [15]). The third study reported that receipt of IIAT significantly increased the multivariable adjusted odds of carbapenem resistant $P$. aeruginosa [14].

\section{Risk factors for acquiring resistant $P$. aeruginosa}

Nineteen studies reported in 17 articles (two articles contributed to four studies) were included (Table 3) [12, 14-16, 18-30]. A total of 3203 total subjects (1077 cases and 2126 controls) were examined for risk factors of acquisition of 
Table 2 Summary baseline table of studies comparing AIAT vs. IIAT

\begin{tabular}{|c|c|c|c|c|c|c|c|c|c|c|c|}
\hline $\begin{array}{l}\text { Author } \\
\text { Year }\end{array}$ & $\begin{array}{l}\text { Country } \\
\text { (Enrollment } \\
\text { Period) }\end{array}$ & $\begin{array}{l}\text { Study } \\
\text { Design }\end{array}$ & $\begin{array}{l}\text { Total } \\
\text { Follow- } \\
\text { up }\end{array}$ & $\begin{array}{l}\text { Total } \\
\mathrm{N}\end{array}$ & Pathogen & Site of Infection & $\begin{array}{l}\text { Mean } \\
\text { Age (SD), } \\
\text { yr }\end{array}$ & $\begin{array}{l}\% \\
\text { Male }\end{array}$ & $\begin{array}{l}\% \\
\text { IIAT }\end{array}$ & $\begin{array}{l}\text { Timeliness } \\
\text { of AIAT }\end{array}$ & $\begin{array}{l}\text { Susceptibility } \\
\text { reported }\end{array}$ \\
\hline $\begin{array}{l}\text { Araujo } \\
2016 \text { [12] }\end{array}$ & $\begin{array}{l}\text { Brazil (2009- } \\
\text { 2012; 2014- } \\
\text { 2014) }\end{array}$ & $\mathrm{CC}$ & $\begin{array}{l}\sim 55 \\
\text { days }\end{array}$ & 236 & $\begin{array}{l}\text { MDR and Non- } \\
\text { MDR PA }\end{array}$ & BSI: $100 \%$ & $\begin{array}{l}52.7 \\
(22.9)\end{array}$ & 70.3 & 36 & $<24 \mathrm{~h}$ & Yes \\
\hline $\begin{array}{l}\text { Dantas } \\
2014 \text { [15] }\end{array}$ & $\begin{array}{l}\text { Brazil (2009- } \\
\text { 2011) }\end{array}$ & $\mathrm{RC}$ & $\begin{array}{l}\sim 55.4 \\
\text { days }\end{array}$ & 120 & $\begin{array}{l}\text { Resistant and } \\
\text { susceptible PA }\end{array}$ & BSI: $100 \%$ & $51.5(3.2)$ & 63.3 & 28.3 & NR & NR \\
\hline $\begin{array}{l}\text { Gonzales } \\
2014 \text { [13] }\end{array}$ & $\begin{array}{l}\text { Colombia } \\
\text { (2005-2008) }\end{array}$ & $\mathrm{RC} ; \mathrm{CC}$ & 30 days & 164 & PA & $\begin{array}{l}\text { RS: } 37.5 \% \text {; Central } \\
\text { venous catheter } \\
28.6 \%\end{array}$ & $56(33.5)$ & 67.1 & 50 & $<48 \mathrm{~h}$ & Yes \\
\hline $\begin{array}{l}\text { Pinheiro } \\
2008 \text { [17] }\end{array}$ & $\begin{array}{l}\text { Brazil (2006- } \\
\text { 2007) }\end{array}$ & $\mathrm{RC} ; \mathrm{CC}$ & 30 days & 131 & PA & $\begin{array}{l}\text { RS: } 65.6 \% \text {; BSI: 18.3\%; } \\
\text { UTI: } 11.5 \%\end{array}$ & $\begin{array}{l}64.2 \\
(18.4)\end{array}$ & 50.4 & 37.3 & NR & Yes \\
\hline $\begin{array}{l}\text { Tuon } \\
2012 \text { [16] }\end{array}$ & $\begin{array}{l}\text { Brazil (2006- } \\
\text { 2009) }\end{array}$ & $\mathrm{CC}$ & 30 days & 77 & CRPA, CSPA & BSI: $100 \%$ & 48.0 & 72.7 & 52 & $<24 h$ & Yes \\
\hline $\begin{array}{l}\text { Rossi } \\
2017 \text { [14] }\end{array}$ & $\begin{array}{l}\text { Brazil (2009- } \\
\text { 2012) }\end{array}$ & CC & $N R$ & 157 & PA $100 \%$ & $\begin{array}{l}\text { Unknown: } 62.42 ; \mathrm{RS}: \\
\text { 17.19; BSI: } 13.37\end{array}$ & $\begin{array}{l}52.0 \\
(24.5)\end{array}$ & 66.9 & 31.2 & NR & Yes \\
\hline
\end{tabular}

AIAT Appropriate initial antibiotic therapy; BSI Bacteremia/Bloodstream infection; CC Case-control; CRPA Carbapenem-resistant Pseudomonas aeruginosa; CSPA Carbapenem-susceptible Pseudomonas aeruginosa; $H$ hour; IIAT Inappropriate initial antibiotic therapy; MDR Multidrug resistant; $N$ Number; NR Not reported; PA Pseudomonas aeruginosa; RC Retrospective; RS Respiratory; UTI Urinary tract infection; Yr Year

MDR and resistant $P$. aeruginosa. Five studies (286 cases of 882 total patients) reported data on MDR P. aeruginosa [12, 18, 19, 22, 30]; 14 studies (12 articles) reported on resistant $P$. aeruginosa (791 cases of 2321 total patients). Included studies were conducted in Brazil (13 studies [12, 14-16, 18, 21, 23-28, 30]), Colombia (3 studies [19, 20, 22]), and Cuba (1 study [29]). Six studies recruited case patients from ICU and the remaining studies included patients admitted to the hospital (Table 3). Risk of bias for these studies is presented in Supplemental Table 4.

The descriptions of study control groups were sparse across studies: susceptible $P$. aeruginosa (8 studies); non- $P$. aeruginosa (1 study); and no specific data was reported in 10 studies. No metaanalysis was performed owing to the lack of common comparator pathogen.

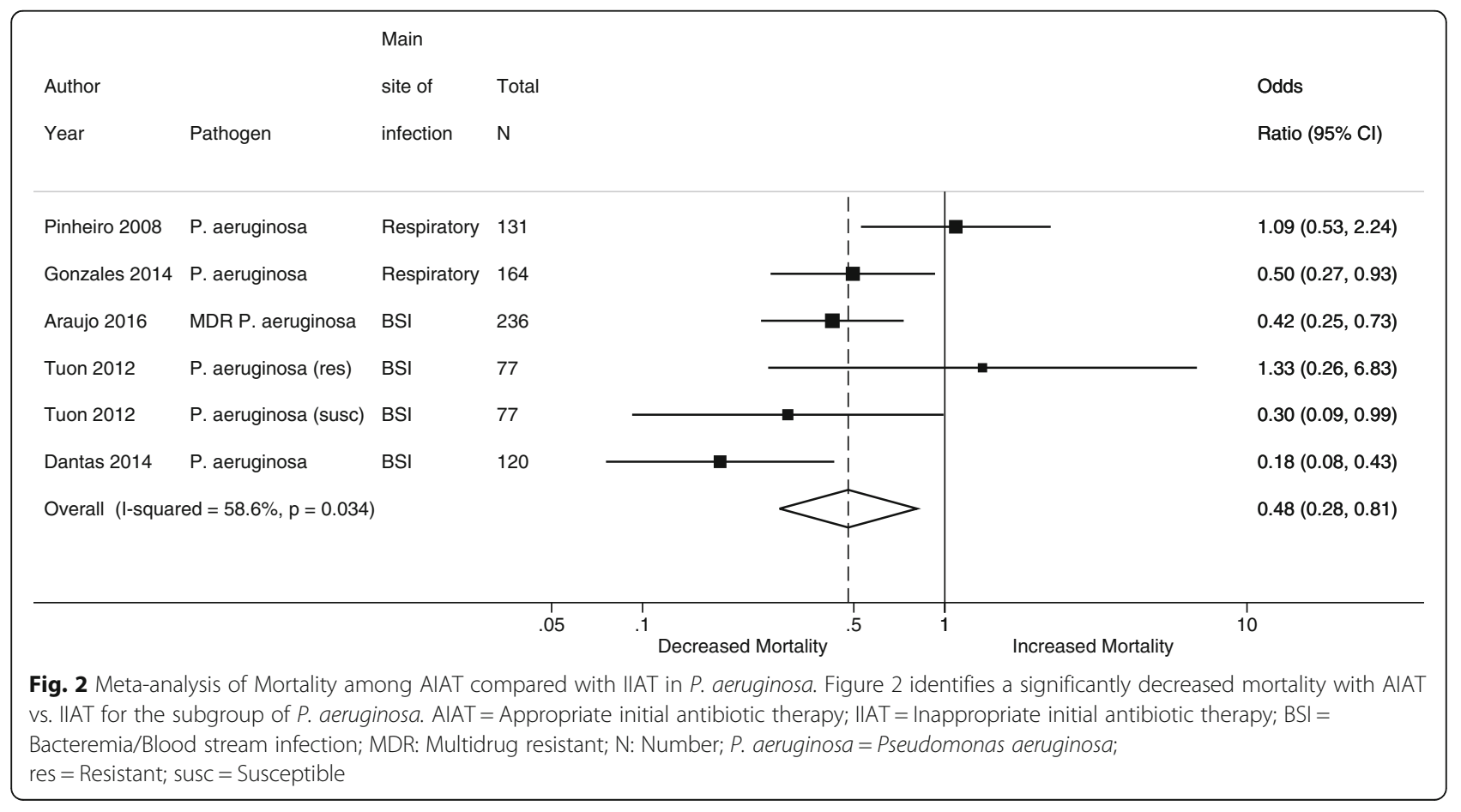


Table 3 Study characteristics and results of risk factors for acquiring $P$. aeruginosa

\begin{tabular}{|c|c|c|c|c|c|c|c|c|c|c|c|}
\hline $\begin{array}{l}\text { Author } \\
\text { Year }\end{array}$ & $\begin{array}{l}\text { Country } \\
\text { (Enrollment } \\
\text { Period) }\end{array}$ & $\begin{array}{l}\text { Study } \\
\text { Design }\end{array}$ & $\begin{array}{l}\text { Hospital } \\
\text { Setting }\end{array}$ & $\begin{array}{l}\text { Type of } \\
\text { Funding }\end{array}$ & $\begin{array}{l}\text { Total } \\
\mathrm{N}\end{array}$ & $\begin{array}{l}\text { Site of } \\
\text { Infection }\end{array}$ & $\begin{array}{l}\text { Source of } \\
\text { infection }\end{array}$ & $\begin{array}{l}\text { Case / } \\
\text { Exposure }\end{array}$ & $\begin{array}{l}\text { Control / } \\
\text { comparator }\end{array}$ & $\begin{array}{l}\text { Mean } \\
\text { Age yr }\end{array}$ & $\begin{array}{l}\% \\
\text { Male }\end{array}$ \\
\hline $\begin{array}{l}\text { Araujo } \\
2016 \text { [12] }\end{array}$ & $\begin{array}{l}\text { Brazil } \\
\text { (2009-2012; } \\
\text { 2014) }\end{array}$ & CC & Academic & Gov & 236 & $\begin{array}{l}\text { Bacteremia } \\
100 \%\end{array}$ & $\begin{array}{l}\text { Nosocomial } \\
\text { and } \\
\text { Community- } \\
\text { acquired }\end{array}$ & MDR-PA & Non-MDR PA & 52.7 & 70.3 \\
\hline $\begin{array}{l}\text { Cortes } 2009 \\
\text { [22] }\end{array}$ & $\begin{array}{l}\text { Colombia } \\
\text { (2001-2002) }\end{array}$ & $\mathrm{CC}$ & Gov & $N R$ & 96 & $\begin{array}{l}\text { Operation } \\
\text { site } 31 \% \text {, } \\
\text { RS } 31 \%, \text { BSI } \\
50 \%\end{array}$ & Nosocomial & MDR-PA & $\begin{array}{l}\text { Random patients } \\
\text { hospitalized the } \\
\text { same day }\end{array}$ & 43.5 & 51 \\
\hline $\begin{array}{l}\text { DalBen } \\
2013 \text { [23] }\end{array}$ & $\begin{array}{l}\text { Brazil } \\
(2000-2002)\end{array}$ & $P C$ & Tertiary & $\begin{array}{l}\text { Gov; } \\
\text { Academic }\end{array}$ & 325 & $N R$ & Nosocomial & CRPA & NA & 44 & 41 \\
\hline $\begin{array}{l}\text { Dantas } \\
2014 \text { [15] }\end{array}$ & $\begin{array}{l}\text { Brazil } \\
(2009-2011)\end{array}$ & $\mathrm{RC}$ & Academic & Gov & 120 & BSI 100\% & NR & $\begin{array}{l}\text { Resistant, } \\
\text { MDR-PA } \\
\text { and XDR- } \\
\text { PA }\end{array}$ & NA & 51.5 & 63.3 \\
\hline $\begin{array}{l}\text { Fortaleza } \\
2006 \text { (Study } \\
\text { 1) }[25]\end{array}$ & $\begin{array}{l}\text { Brazil } \\
(1992-2002)\end{array}$ & $\mathrm{CC}$ & Academic & $N R$ & 324 & $\begin{array}{l}\text { Wound } \\
21.3 \%, \mathrm{BSI} \\
19.4 \% \text {, UTI } \\
16.7 \%\end{array}$ & Nosocomial & $\begin{array}{l}\text { IRPA or } \\
\text { Ceftazidine- } \\
\text { resistant PA }\end{array}$ & $\begin{array}{l}\text { Patients without } \\
\text { IRPA who were } \\
\text { admitted to the } \\
\text { same ward }\end{array}$ & 44.3 & 63 \\
\hline $\begin{array}{l}\text { Fortaleza } \\
2006 \text { (Study } \\
\text { 2) [25] }\end{array}$ & $\begin{array}{l}\text { Brazil } \\
(1992-2002)\end{array}$ & $\mathrm{CC}$ & Academic & $N R$ & 165 & $\begin{array}{l}\text { UTI 27.3\%, } \\
\text { RS 25.5\%, } \\
\text { wounds } \\
21.8 \%\end{array}$ & NR & $\begin{array}{l}\text { IRPA or } \\
\text { Ceftazidine- } \\
\text { resistant PA }\end{array}$ & $\begin{array}{l}\text { Patients without } \\
\text { IRPA who were } \\
\text { admitted to the } \\
\text { same ward }\end{array}$ & 42.3 & 66 \\
\hline $\begin{array}{l}\text { Furtado } \\
2009 \text { [28] }\end{array}$ & $\begin{array}{l}\text { Brazil } \\
(2003-2004)\end{array}$ & $\mathrm{CC}$ & Academic & $N R$ & 245 & $\begin{array}{l}\text { UTI 34.9\%; } \\
\text { RS 22.2\%; } \\
\text { catheter tip } \\
20.6 \%\end{array}$ & Nosocomial & IRPA & $\begin{array}{l}\text { Patients } \\
\text { hospitalized in the } \\
\text { same unit and } \\
\text { matched to case } \\
\text { patients }\end{array}$ & $\begin{array}{l}\text { Median: } \\
\text { Cases: } \\
50, \\
\text { Controls: } \\
54\end{array}$ & 61.6 \\
\hline $\begin{array}{l}\text { Furtado } \\
2010 \text { [24] }\end{array}$ & $\begin{array}{l}\text { Brazil } \\
(2006-2008)\end{array}$ & CC & Academic & $N R$ & 295 & RS 100\% & Nosocomial & IRPA & $\begin{array}{l}\text { Patients without } \\
\text { PA receiving care } \\
\text { in same ICU }\end{array}$ & 54 & 59.3 \\
\hline $\begin{array}{l}\text { Gomes } \\
2012 \text { [30] }\end{array}$ & $\begin{array}{l}\text { Brazil } \\
(2002-2007)\end{array}$ & PCC & Tertiary & Gov & 60 & NR & Nosocomial & MDR-PA & Controls & $\begin{array}{l}\text { Median: } \\
\text { Cases: } \\
\text { 50, } \\
\text { Controls: } \\
40\end{array}$ & 66.7 \\
\hline $\begin{array}{l}\text { Medell } \\
2012 \text { [29] }\end{array}$ & Cuba (2011) & $P C$ & Tertiary & Gov & 12 & VAP $100 \%$ & Nosocomial & PA & NA & 55.5 & $N R$ \\
\hline $\begin{array}{l}\text { Neves } 2010 \\
\text { [18] }\end{array}$ & $\begin{array}{l}\text { Brazil } \\
(2004-2005)\end{array}$ & $\begin{array}{l}\text { Ecological } \\
\text { design; RC }\end{array}$ & Academic & $N R$ & 350 & NR & NR & MDR-PA & NA & $N R$ & NR \\
\hline $\begin{array}{l}\text { Ossa- } \\
\text { Giraldo } \\
2014 \text { [19] }\end{array}$ & $\begin{array}{l}\text { Colombia } \\
\text { (2009-2010) }\end{array}$ & $C C$ & Academic & $\begin{array}{l}\text { University } \\
\text { Hospital }\end{array}$ & 140 & $N R$ & Nosocomial & MDR-PA & Susceptible PA & 43.3 & 70 \\
\hline $\begin{array}{l}\text { Pereira } \\
2008 \text { [27] }\end{array}$ & $\begin{array}{l}\text { Brazil } \\
(2000-2002)\end{array}$ & $\mathrm{CC}$ & Academic & Academic & 59 & $\begin{array}{l}\text { UTI 60\%, } \\
\text { BSI 7\%, RS } \\
17 \%\end{array}$ & Nosocomial & IRPA & ISPA & 51.3 & 62.7 \\
\hline $\begin{array}{l}\text { Rossi } 2017 \\
{[14]}\end{array}$ & $\begin{array}{l}\text { Brazil } \\
(2009-2012)\end{array}$ & $\mathrm{CC}$ & Academic & $\begin{array}{l}\text { University } \\
\text { Hospital }\end{array}$ & 157 & $\begin{array}{l}\text { Unknown: } \\
\text { 62.42; RS: } \\
\text { 17.19; BSI: } \\
13.37\end{array}$ & Nosocomial & CRPA & CSPA & 66.9 & 31.2 \\
\hline $\begin{array}{l}\text { Royer } 2015 \\
\text { [21] }\end{array}$ & $\begin{array}{l}\text { Brazil } \\
(2011-2012)\end{array}$ & PC & Academic & Gov & 30 & VAP $100 \%$ & Nosocomial & CRPA & NA & 58.97 & 80 \\
\hline $\begin{array}{l}\text { Tuon } 2012 \\
\text { [16] }\end{array}$ & $\begin{array}{l}\text { Brazil } \\
(2006-2009)\end{array}$ & CC & Tertiary & $N R$ & 77 & BSI 100\% & Nosocomial & CRPA & CSPA & 47.4 & 23.7 \\
\hline $\begin{array}{l}\text { Valderrama } \\
2016 \text { [20] }\end{array}$ & $\begin{array}{l}\text { Colombia } \\
(2008-2014)\end{array}$ & CC & Academic & $\begin{array}{l}\text { University } \\
\text { Hospital }\end{array}$ & 168 & $\begin{array}{l}\text { RS 30\%; GI } \\
26 \% \text {; } \\
\text { Primary } \\
13.7 \%\end{array}$ & Nosocomial & CRPA & CSPA & $\begin{array}{l}\text { Cases: } \\
60 ; \\
\text { Controls: } \\
64.5\end{array}$ & 53 \\
\hline
\end{tabular}


Table 3 Study characteristics and results of risk factors for acquiring $P$. aeruginosa (Continued)

\begin{tabular}{|c|c|c|c|c|c|c|c|c|c|c|c|}
\hline $\begin{array}{l}\text { Author } \\
\text { Year }\end{array}$ & $\begin{array}{l}\text { Country } \\
\text { (Enrollment } \\
\text { Period) }\end{array}$ & $\begin{array}{l}\text { Study } \\
\text { Design }\end{array}$ & $\begin{array}{l}\text { Hospital } \\
\text { Setting }\end{array}$ & $\begin{array}{l}\text { Type of } \\
\text { Funding }\end{array}$ & $\begin{array}{l}\text { Total } \\
\mathrm{N}\end{array}$ & $\begin{array}{l}\text { Site of } \\
\text { Infection }\end{array}$ & $\begin{array}{l}\text { Source of } \\
\text { infection }\end{array}$ & $\begin{array}{l}\text { Case / } \\
\text { Exposure }\end{array}$ & $\begin{array}{l}\text { Control / } \\
\text { comparator }\end{array}$ & $\begin{array}{l}\text { Mean } \\
\text { Age yr }\end{array}$ & $\begin{array}{l}\% \\
\text { Male }\end{array}$ \\
\hline $\begin{array}{l}\text { Zavascki } \\
2005 \\
\text { (Study 1) } \\
\text { [26] }\end{array}$ & $\begin{array}{l}\text { Brazil } \\
(2002-2003)\end{array}$ & CC & Tertiary & Gov & 186 & $\begin{array}{l}\text { IRPA:RS } \\
\text { 33.4\%, UTI } \\
\text { 26.9\%, } \\
\text { Control: NR }\end{array}$ & Nosocomial & IRPA & $\begin{array}{l}\text { Random patients } \\
\text { from same unit }\end{array}$ & 54.5 & 56.5 \\
\hline $\begin{array}{l}\text { Zavascki } \\
2005 \\
\text { (Study2) } \\
{[26]}\end{array}$ & $\begin{array}{l}\text { Brazil } \\
(2002-2003)\end{array}$ & $\mathrm{CC}$ & Tertiary & Gov & 158 & $\begin{array}{l}\text { IRPA: RS } \\
\text { 33.4\%, UTI } \\
\text { 26.9\%, } \\
\text { Control: NR }\end{array}$ & Nosocomial & IRPA & ISPA & 54.7 & 62.7 \\
\hline
\end{tabular}

BSI Bacteremia; CC Case-control; CRPA Carbapenem resistant Pseudomonas aeruginosa; CSPA Carbapenem susceptible Pseudomonas aeruginosa; Gov Government; GI Gastrointestinal; IRPA imipenem-resistant Pseudomonas aeruginosa; ISPA imipenem- susceptible Pseudomonas aeruginosa; MDR Multi-drug resistant; N Number; NA Not applicable; NR Not reported; PA Pseudomonas aeruginosa; PC Prospective; PCC Prospective case-control; RC Retrospective; $R S$ Respiratory; UTI Urinary tract infection; VAP ventilator-associated pneumonia; $X D R$ Extreme drug resistant; $Y r$ year

\section{Results of studies of MDR P. aeruginosa}

All five studies of MDR P. aeruginosa examined prior use of antibiotics, two studies examined ICU stay, and one study each examined a variety of risk factors: co-occurring disease, surgical procedure, hospital stay, inappropriate therapy, enteral feeding, parenteral feeding, mechanical ventilation, and female sex (Table 4). We did not perform meta-analysis owing to the small number of eligible studies for any particular comparison.

Two studies each reported that previous use of aminoglycoside, carbapenem, and fluorquinolones was a significant predictor for acquisition of MDR P. aeruginosa. In one study, all variables were significant (Table 4), except for the presence of type II diabetes, undergoing surgical procedure, and ICU stay.

\section{Results of studies of Resistant $P$. aeruginosa}

Of 14 studies in 12 articles, the significant predictors of acquisition of resistant $P$. aeruginosa were prior use of antibiotics (9 studies), stay in the ICU (3 studies), and comorbidity score (3 studies). Significant predictors were reported in at least two studies for each of the following: co-occurring diseases, hospital stay, hemodialysis, length of stay, male sex, and parenteral nutrition. Other significant predictors reported in at least one study included: mechanical ventilation, inappropriate therapy, colonization pressure, admission diagnosis, surgical procedure, use of corticosteroids, respiratory infection, and transfer from another hospital.

Nine studies (in 11 data points) reported prior use of different classes of antibiotics as a significant predictor for acquisition of resistant $P$. aeruginosa. The most frequently assessed antibiotics included carbapenem and aminoglycosides (Supplemental Table 5). All of the examined predictors were reported as significant predictors for acquisition of resistant $P$. aeruginosa except for the following in three studies: Acute Physiology And Chronic Health Evaluation II (APACHE II) and hospital stay (1 study [20]), heart failure as a co-occurring disease (1 study [21]), and number of antibiotics used (1 study [24]).

\section{GRADE rating of overall strength of evidence}

The overall GRADE rating showed that there was low strength of evidence across outcomes that were primary and/or most frequently reported in eligible studies (Supplemental Table 6), These included mortality for the comparisons of AIAT with IIAT (primary outcome), and the most frequently reported outcomes of previous use of antibiotics, comorbidity score, and hospital stay as a risk factor for the acquisition of MDR or resistant $P$. aeruginosa, and hospital stay and APACHE II as a risk factor for the acquisition of resistant $P$. aeruginosa. All the aforementioned outcomes were graded to be of low strength of evidence owing to risk of bias (unclear or high) and imprecise estimates.

\section{Discussion}

This systematic literature review and meta-analysis of studies from the LATAM region identified several important findings among hospitalized adult patients with $P$. aeruginosa. First, AIAT compared with IIAT was associated with a halving of 30-day mortality (unadjusted summary $\mathrm{OR}=0.48$ ). This significance persisted in subgroup analyses, when appropriate antibiotics were initiated within $48 \mathrm{~h}$, in blood stream infections, and among patients admitted to the hospital wards. Although there were no studies to draw conclusions for $<24-\mathrm{h}$ or 24 -to-48-h time points, this review demonstrates that a 48$\mathrm{h}$ time point is a possible threshold beyond which a significantly higher mortality risk is observed with further delays in AIAT.

Second, the choice of an initial antibiotic therapy against a possible $P$. aeruginosa infection has been a challenge owing, in part, to different resistance trends, mechanisms of resistance and evolution of resistance. For example, resistance to the carbapenem class, an important class of initial therapy in severe nosocomial infections, is now reported to be more than $70 \%$ in some hospitals in the LATAM region [32]. Indeed, a number of individual studies exist in the public domain 
Table 4 Risk for Predicting Acquisition of Multi-drug Resistant Pseudomonas aeruginosa

\begin{tabular}{|c|c|c|c|c|c|c|}
\hline Risk Category & Risk Factor & Author Year & $\begin{array}{l}\mathrm{N} \\
\text { MDRPA }\end{array}$ & $\begin{array}{l}\mathrm{N} \\
\text { Control }\end{array}$ & $\begin{array}{l}\text { Control } \\
\text { description }\end{array}$ & $\begin{array}{l}\text { Multivariate Results (95\%LCl, } \\
\text { 95\%UCl); } \boldsymbol{P} \text {-value }\end{array}$ \\
\hline $\begin{array}{l}\text { Co-occurring } \\
\text { condition }\end{array}$ & Diabetes Mellitus & Araujo 2016 [12] & 96 & 140 & Non-MDR PA & OR $1.9907(0.97,4.09) ; 0.0608$ \\
\hline \multirow[t]{2}{*}{ Procedure } & Surgery & Cortes 2009 [22] & 24 & 72 & Control & OR $2.8(0.7,11.7) ; 0.14$ \\
\hline & Mechanical ventilation & & & & & OR $12.2(0.1,12.6) ; 0.014$ \\
\hline \multirow[t]{3}{*}{ Stay } & Hospital & $\begin{array}{l}\text { Ossa-Giraldo } 2014 \\
\text { [19] }\end{array}$ & 70 & 70 & Susceptible PA & OR 1.03 (1.01,1.05); sig \\
\hline & ICU & Cortes 2009 [22] & 24 & 72 & Control & OR $1.2(0.6,12.7) ; 0.91$ \\
\hline & ICU & Dantas 2014 $[15]$ & 57 & 65 & Resistant PA & OR 3.28 (NR); 0.02 \\
\hline Therapy & \|AT & Araujo 2016 [12] & 96 & 140 & Non-MDR PA & OR $3.0169(1.72,5.31) ; 0.0001$ \\
\hline \multirow[t]{2}{*}{ Feeding } & Parenteral feeding & Gomes 2012 [30] & 15 & 45 & Non-MDR PA & OR 10.7 (1.5,91.9); 0.018 \\
\hline & Enteral feeding & Gomes 2012 [30] & 15 & 45 & Non-MDR PA & OR $14.9(3.3,94.1) ; 0.003$ \\
\hline \multirow[t]{9}{*}{$\begin{array}{l}\text { Prior antibiotic } \\
\text { use }\end{array}$} & Aminoglycoside & Neves 2010 [18] & 81 & 269 & Non-MDR PA & $\begin{array}{l}\text { Correlation coefficient } 0.31 \text { (NR); } \\
0.14\end{array}$ \\
\hline & Aminoglycoside & $\begin{array}{l}\text { Ossa-Giraldo } 2014 \\
\text { [19] }\end{array}$ & 70 & 70 & Susceptible PA & OR 3.09 (1.26,7.58); NR \\
\hline & Carbapenem & Neves 2010 [18] & 81 & 269 & Non-MDR PA & $\begin{array}{l}\text { Correlation coefficient } 0.67 \text { (NR); } \\
0.01\end{array}$ \\
\hline & Carbapenem & Araujo 2016 [12] & 96 & 140 & Non-MDR PA & OR $0.8928(0.51,1.55) ; 0.6873$ \\
\hline & Quinolone & Neves 2010 [18] & 81 & 269 & Non-MDR PA & $\begin{array}{l}\text { Correlation coefficient }-0.13 \text { (NR); } \\
0.57\end{array}$ \\
\hline & Quinolone & Gomes 2012 [30] & 15 & 45 & Non-MDR PA & OR $8.9(1.6,66.4) ; 0.013$ \\
\hline & 3rd-generation Cephalosporin & Neves 2010 [18] & 81 & 269 & Non-MDR PA & $\begin{array}{l}\text { Correlation coefficient } 0.16 \text { (NR); } \\
0.31\end{array}$ \\
\hline & $\begin{array}{l}>2 \text { antimicrobials for }>48 \mathrm{~h} \text { in } \\
\text { prior } 30 \mathrm{~d}\end{array}$ & $\begin{array}{l}\text { Ossa-Giraldo } 2014 \\
\text { [19] }\end{array}$ & 70 & 70 & Susceptible PA & OR 4.4 (1.1,17.65); NR \\
\hline & Any & Cortes 2009 [22] & 24 & 72 & Control & OR $2.7(1.2125) ; 0.19$ \\
\hline Sex & Female & $\begin{array}{l}\text { Ossa-Giraldo } 2014 \\
\text { [19] }\end{array}$ & 70 & 70 & Susceptible PA & OR 2.31 (1.02,5.2); sig \\
\hline $\begin{array}{l}\text { Source of } \\
\text { infection }\end{array}$ & $\begin{array}{l}\text { Respiratory tract source of } \\
\text { bacteremia }\end{array}$ & Dantas 2014 & 57 & 65 & Resistant PA & 5.83 (NR); 0.02 \\
\hline
\end{tabular}

a Both MDRPA and XDRPA

ICU Intensive care unit; LCI Lower confidence interval; MDRPA Multi-drug Resistant; N Number; NR Not reported; OR Odds ratio; PA Pseudomonas aeruginosa; UCI Upper confidence interval

examining risk factors associated with resistant $P$. aeruginosa infections in LATAM. Notably, prior use of antibiotics (11 studies), stay in the ICU (3 studies), and comorbidity score (3 studies) were significant predictors of acquisition of resistant $P$. aeruginosa.

The mechanisms of resistance and variation in resistance based on the type of antibiotics can present some complexities in targeting antibiotic therapy involving either acquisition of genes against betalactams and aminoglycosides, or mutation of chromosomal genes in the case of resistance against fluoroquinolones [33]. These challenges may result in delays in the initiation and administration of appropriate antibiotics that can lead to adverse patient outcomes. An understanding of local epidemiology specific to the LATAM region is essential in early diagnosis and administration of AIAT.
The development of resistance can also evolve over time. For example, $P$. aeruginosa has evolved virulence characteristics that may make it a difficult target for antibiotic therapy. Resistant strains of $P$. aeruginosa are the most frequent source of HAIs and are often associated with overuse or inappropriate use of antimicrobials. Our review highlights that there was a consistent association between prior use of antimicrobials and acquisition of resistant $P$. aeruginosa. Our findings concur with many other studies that showed exposure to previous antimicrobial therapy prior to the development of current infection can lead an increased risk of resistance to different classes of antibiotics [34]. A thorough understanding of risk factors associated with acquisition of resistant $P$. aeruginosa can aid in the monitoring of resistant pathogens in order to prevent emergence of MDR/XDR/pan-drug resistant pathogens. More 
importantly, knowledge about these risk factors can help tailor presumptively better antimicrobials to be used.

This review and meta-analysis have several strengths. To the best of our knowledge, there was no previously published systematic review and meta-analysis from the LATAM region comparing AIAT with IIAT and systematically reviewed studies from the LATAM region evaluating multivariate risk factors predicting acquisition of $P$. aeruginosa. This review included studies published in English, Spanish and Portuguese languages from the LATAM region. Additionally, this review comprehensively searched for studies indexed in regional databases. The overall results from this review are consistent with other systematic reviews published literature from other regions that compare the role of AIAT with IIAT in mortality $[8,35,36]$. Nonetheless, in light of a few limitations, the results of the systematic review and metaanalysis should be interpreted with caution. There was a general lack of studies reporting adjusted analyses for mortality outcomes. Therefore, our meta-analysis is restricted to unadjusted results comparing AIAT with IIAT that did not account for potential confounders. Overall, risk of bias among individual studies was high and there was inconsistencies across studies. The majority of studies were retrospective observational studies. This translated into a low overall strength of evidence that was confirmed by a formal evaluation of the GRADE to assess the overall strength of evidence. The impact of AIAT versus IIAT on treatment response, length of stay, and costs in the LATAM region is unknown because there were no studies evaluating these outcomes. In addition, studies either did not clearly define AIAT and IIAT, or lacked consistent definitions on timeliness and susceptibility to antimicrobial therapy.

The following gaps in the literature merit consideration for future research. There is a need to examine outcome measures including costs, length of stay, clinical and microbiologic success in studies published from the LATAM region in future studies. Data on the risk factors predicting acquisition of $P$. aeruginosa were generally inconsistent or not particularly highlighting any particular factors. The limitations of this review as well as any future reviews will reflect, to a large extent, the limitations of the data in primary studies. Therefore, future, well-conducted, well-analyzed (preferably prospective) observational studies are warranted in this area.

\section{Conclusion}

This systematic literature review and meta-analysis suggests significantly decreased 30-day mortality with AIAT, as compared with IIAT among patients hospitalized with $P$. aeruginosa infections in the LATAM region. This review also highlights that use of prior antibiotics, co-morbid diseases or severity scores, and prior ICU/ hospital stay as consistent and significant risk predictors of acquisition of resistant $P$. aeruginosa among hospitalized patients from the LATAM region.

This review synthesizes important evidence from the LATAM region, which to the best of our knowledge, was not systematically assessed before. This evidence that may make a significant impact on clinical and health policy decision-making. The policy implementation of our findings in the LATAM region would benefit by engaging infectious disease specialists, hospitalists, epidemiologists, and other relevant stakeholders. AIAT against a possible infection has been a challenge owing, in part, to different resistance trends in several regions of the world. In addition to implementation of infection control programs in hospitals, a thorough understanding of local epidemiology and better laboratory facilities are essential in targeting appropriate antibiotic. Given the high resistance rates in the LATAM region and the worse outcomes associated with $P$. aeruginosa infections, there is a continued need for the use of appropriate antimicrobial agents with activity against $P$. aeruginosa. The consistent role of prior use of antibiotics or a stay in ICU in the acquisition of resistant $P$. aeruginosa reinforces the importance of antimicrobial stewardship programs, especially in the ICU setting among critically ill patients.

\section{Supplementary information}

Supplementary information accompanies this paper at https://doi.org/10. 1186/s12879-020-04973-0.

Additional file 1 Table $\mathbf{S 1}$. Search Strategy comparing AIAT VS. IIAT Table S2. Search Strategy for acquisition of resistant $P$. aeruginosa. Table S3. Risk of Bias Assessment for AIAT vs. IIAT Studies. Table S4. Risk of Bias Assessment for Risk Factors Studies. Table S5. Risk Factors Predicting Acquisition of Resistant P. aeruginosa. Table S6. GRADE Overall Strength of Evidence for Relevant Outcomes.

\section{Abbrevations}

AHRQ: Agency for Healthcare Research and Quality; AIAT: Appropriate initial antibiotic therapy; APACHE II: Acute Physiology And Chronic Health Evaluation II; BIREME: Biblioteca Regional de Medicina; Cl: Confidence Interval; ES: Effect size; ESBL: Extended-spectrum beta-lactamases; GRADE: Grading of Recommendations Assessment, Development and Evaluation; HAls: Healthcare associated infections; HR: Hazard Ratio; ICU: Intensive Care Unit; IIAT: Inappropriate initial antibiotic therapy; LATAM: Latin American countries; LILAC: Literatura Latino Americana em Ciências da Saúde; MDR: Multi-drug Resistant; NA: Not applicable; OR: Odds Ratio; PACTS: Program to Assess Ceftolozane/Tazobactam Susceptibility; PRISMA: Preferred Reporting Items for Systematic Reviews and MetaAnalyses; XDR: Extremely drug-resistant

\section{Acknowledgements}

Not applicable.

\section{Authors' contributions}

APdL, SM, GTH, ES. designed research; GR, EEA, and JC conducted research; GR and EEA analyzed data; and GR, EEA, and JC wrote the paper; APdL, SM, GTH, ES edited the paper; GR, EEA, and JC. had primary responsibility for final content. All authors read and approved the final manuscript. 


\section{Funding}

Funding for this review was provided to Tufts Medical Center by Merck Sharp \& Dohme Corp., a subsidiary of Merck \& Co., Inc., Kenilworth, NJ, USA The funder played no role in the study search, selection, and analysis that were conducted by researchers at the Tufts Medical Center.

\section{Availability of data and materials}

Data supporting the conclusions of this article is available in the Supplementary Material, figures, and tables. Detailed data extraction forms are available upon request from the corresponding author.

\section{Ethics approval and consent to participate}

Not applicable because this is a review of published literature.

\section{Consent for publication}

Not applicable.

\section{Competing interests}

S. Merchant, G. T. Hernandez, and E. Sarpong are employees of Merck Sharp \& Dohme Corp., a subsidiary of Merck \& Co., Inc., Kenilworth, NJ, USA.

\section{Author details}

${ }^{1}$ Department of Infectious Diseases, Laboratory of Clinical Microbiology, Instituto Nacional de Ciencias Médicas y Nutrición Salvador Zubirán, Mexico City, Mexico. ${ }^{2}$ Merck \& Co., Inc, Kenilworth, NJ, USA. ${ }^{3}$ Institute for Clinical Research and Health Policy Studies, Center for Clinical Evidence Synthesis, Tufts Medical Center, 800 Washington St, Boston, MA 02111, USA. ${ }^{4}$ Tufts University School of Medicine, Boston, MA, USA.

\section{Received: 28 June 2019 Accepted: 13 March 2020}

Published online: 27 March 2020

\section{References}

1. Antibiotic resistance threats in the United States, 2013. Atlanta, Center for Disease Control. http://www cdc gov/drugresistance/threat-report-2013/pdf/ar-threats2013-508 pdf 2013;Available from: URL: http://www.cdc.gov/drugresistance/ threat-report-2013/pdf/ar-threats-2013-508.pdf [cited 2017 May 15].

2. Antimicrobial Resistance Global report on surveillance. Genevä, World Health Organization. http://apps who int/iris/bitstream/10665/112642/1/ 9789241564748_eng pdf 2014;Available from: URL: http://apps.who.int/iris/ bitstream/10665/112642/1/9789241564748_eng.pdf [cited 2017 May 15].

3. Farrell DJ, Sader HS, Flamm RK, Jones RN. Frequency of occurrence and antimicrobial susceptibility of Gram-negative organisms isolated from health care-associated (HCA) urinary tract infections (UTI) in the United States: Results from the Program to Assess Ceftolozane/Tazobactam Susceptibility (PACTS). Open Forum Infect Dis. 2014;1(suppl_1):S105-6.

4. Sader HS, Farrell DJ, Flamm RK, Jones RN. Ceftolozane/tazobactam activity tested against aerobic gram-negative organisms isolated from intraabdominal and urinary tract infections in European and United States hospitals (2012). J Inf Secur. 2014;69(3):266-77.

5. Zilberberg MD, Shorr AF, Micek ST, Vazquez-Guillamet C, Kollef MH. Multidrug resistance, inappropriate initial antibiotic therapy and mortality in gram-negative severe sepsis and septic shock: a retrospective cohort study. Crit Care. 2014;18(6):596.

6. Goff DA, Kullar R, Bauer KA, File TM Jr. Eight habits of highly effective antimicrobial stewardship programs to meet the joint commission standards for hospitals. Clin Infect Dis. 2017;64(8):1134-9.

7. Moher D, Liberati A, Tetzlaff J, Altman DG. Preferred reporting items for systematic reviews and meta-analyses: the PRISMA statement. Ann Intern Med. 2009;151(4):264-9 W64.

8. Raman G, Avendano E, Berger S, Menon V. Appropriate initial antibiotic therapy in hospitalized patients with gram-negative infections: systematic review and meta-analysis. BMC Infect Dis. 2015;15:395.

9. Methods Guide for Effectiveness and Comparative Effectiveness Review. AHRQ Publication No 10(11)-EHC063-EF. 2011. Chapters Available at: http:// effectivehealthcare.ahrq.gov. Accessed 20 Jan 2020

10. DerSimonian R, Laird N. Meta-analysis in clinical trials. Control Clin Trials. 1986 Sep;7(3):177-88.

11. Borenstein M, Higgins JP, Hedges LV, Rothstein HR. Basics of meta-analysis: I(2) is not an absolute measure of heterogeneity. Res Synth Methods. 2017 Mar;8(1):5-18.
12. Araujo BF, Ferreira ML, Campos PA, Royer S, Batistao DW, Dantas RC, et al. Clinical and Molecular Epidemiology of Multidrug-Resistant $P$. aeruginosa Carrying aac(6')-lb-cr, qnrS1 and blaSPM Genes in Brazil. PLoS ONE [Electronic Resource]. 2016:11(5):e0155914.

13. Gonzalez AL, Leal AL, Cortes JA, Sanchez R, Barrero LI, Castillo JS, et al. [Effect of adequate initial antimicrobial therapy on mortality in critical patients with Pseudomonas aeruginosa bacteremia]. [Spanish]. Biomedica. 2014;349(Suppl):58-66

14. Rossi Gl, Dantas RC, Ferreira ML, Batistao DW, Gontijo-Filho PP, Ribas RM. Carbapenem-resistant Pseudomonas aeruginosa: association with virulence genes and biofilm formation. Braz J Microbiol. 2017 Apr;48(2):211-7.

15. Dantas RC, Ferreira ML, Gontijo-Filho PP, Ribas RM. Pseudomonas aeruginosa bacteraemia: independent risk factors for mortality and impact of resistance on outcome. J Med Microbiol. 2014;63(Pt:12):12-87.

16. Tuon FF, Gortz LW, Rocha JL. Risk factors for pan-resistant Pseudomonas aeruginosa bacteremia and the adequacy of antibiotic therapy. Braz J Infect Dis. 2012;16(4):351-6.

17. Pinheiro MR, Lacerda HR, Melo RG, Maciel MA. Pseudomonas aeruginosa infections: factors relating to mortality with emphasis on resistance pattern and antimicrobial treatment. Braz J Infect Dis. 2008;12(6):509-15.

18. Neves MT, Lorenzo ME, Almeida RA, Fortaleza CM. Antimicrobial use and incidence of multidrug-resistant Pseudomonas aeruginosa in a teaching hospital: an ecological approach. Rev Soc Bras Med Trop. 2010;43(6):629-32.

19. Ossa-Giraldo AC, Echeverri-Toro LM, Santos ZM, Garcia MG, Agudelo Y, Ramirez F, et al. Risk factors for multidrug-resistant Pseudomonas aeruginosa infection, in a tertiary hospital in Colombia. Rev Chil Infectol. 2014;31(4):393-9.

20. Valderrama SL, Gonzalez PF, Caro MA, Ardila N, Ariza B, Gil F, et al. Risk factors for hospital-acquired bacteremia due to carbapenem-resistant Pseudomonas aeruginosa in a Colombian hospital. Biomedica. 2016:36(0):69-77.

21. Royer S, Faria AL, Seki LM, Chagas TP, Campos PA, Batistao DW, et al. Spread of multidrug-resistant Acinetobacter baumannii and Pseudomonas aeruginosa clones in patients with ventilator-associated pneumonia in an adult intensive care unit at a university hospital. Braz J Infect Dis. 2015;19(4):350-7.

22. Cortes JA, Cuervo SI, Urdaneta AM, Potdevin G, Arroyo P, Bermudez D, et al. Identifying and controlling a multiresistant Pseudomonas aeruginosa outbreak in a Latin-American cancer Centre and its associated risk factors. Braz J Infect Dis. 2009:13(2):99-103.

23. DalBen MF, Basso M, Garcia CP, Costa SF, Toscano CM, Jarvis WR, et al. Colonization pressure as a risk factor for colonization by multiresistant Acinetobacter spp and carbapenem-resistant Pseudomonas aeruginosa in an intensive care unit. Clinics (Sao Paulo). 2013;68(8):1128-33.

24. Furtado GH, Gales AC, Perdiz LB, Santos AE, Wey SB, Medeiros EA. Risk factors for hospital-acquired pneumonia caused by imipenem-resistant Pseudomonas aeruginosa in an intensive care unit. Anaesth Intensive Care. 2010;38(6):994-1001

25. Fortaleza CM, Freire MP, Filho DC, de Carvalho RM. Risk factors for recovery of imipenem- or ceftazidime-resistant pseudomonas aeruginosa among patients admitted to a teaching hospital in Brazil. Infect Control Hosp Epidemiol. 2006;27(9):901-6.

26. Zavascki AP, Cruz RP, Goldani LZ. Risk factors for imipenem-resistant Pseudomonas aeruginosa: a comparative analysis of two case-control studies in hospitalized patients. J Hosp Infect. 2005:59(2):96-101.

27. Pereira GH, Levin AS, Oliveira HB, Moretti ML. Controlling the clonal spread of Pseudomonas aeruginosa infection. Infect Control Hosp Epidemiol. 2008;29(6):549-52.

28. Furtado $\mathrm{GH}$, Bergamasco MD, Menezes FG, Marques D, Silva A, Perdiz LB, et al. Imipenem-resistant Pseudomonas aeruginosa infection at a medical-surgical intensive care unit: risk factors and mortality. J Crit Care. 2009;24(4):625-14.

29. Medell M, Hart M, Marrero O, Espinosa F, de OZ M, Valdes R. Clinical and microbiological characterization of pneumonia in mechanically ventilated patients. Braz J Infect Dis. 2012;16(5):442-7.

30. Gomes MZ, Oliveira RV, Machado CR, Conceicao MS, Souza CV, Lourenco $M C$, et al. Factors associated with epidemic multiresistant Pseudomonas aeruginosa infections in a hospital with AIDS-predominant admissions. Braz J Infect Dis. 2012;16(3):219-25.

31. Tuon FF, Graf ME, Merlini A, Rocha UL, Stallbaum S, Arend LN, et al. Risk factors for mortality in patients with ventilator-associated pneumonia caused by carbapenemresistant Enterobacteriaceae. Braz J Infect Dis. 2017;21(1):1-6.

32. Baumgart AM, Molinari MA, Silveira AC. Prevalence of carbapenem resistant Pseudomonas aeruginosa and Acinetobacter baumannii in high complexity hospital. Braz J Infect Dis. 2010;14(5):433-6. 
33. Livermore DM. Multiple mechanisms of antimicrobial resistance in Pseudomonas aeruginosa: our worst nightmare? Clin Infect Dis. 2002;34(5):634-40.

34. Pena C, Suarez C, Gozalo M, Murillas J, Almirante B, Pomar V, et al. Prospective multicenter study of the impact of carbapenem resistance on mortality in Pseudomonas aeruginosa bloodstream infections. Antimicrob Agents Chemother. 2012;56(3):1265-72.

35. Merchant S, Proudfoot EM, Quadri HN, McElroy HJ, Wright WR, Gupta A, et al. Risk factors for Pseudomonas aeruginosa infections in Asia-Pacific and consequences of inappropriate initial antimicrobial therapy: a systematic literature review and meta-analysis. J Glob Antimicrob Resist. 2018;14:33-44.

36. Raman G, Avendano EE, Chan J, Merchant S, Puzniak L. Risk factors for hospitalized patients with resistant or multidrug-resistant Pseudomonas aeruginosa infections: a systematic review and meta-analysis. Antimicrob Resist Infect Control. 2018;7:79. https://doi.org/10.1186/s13756-018-0370-9.

\section{Publisher's Note}

Springer Nature remains neutral with regard to jurisdictional claims in published maps and institutional affiliations.

Ready to submit your research? Choose BMC and benefit from:

- fast, convenient online submission

- thorough peer review by experienced researchers in your field

- rapid publication on acceptance

- support for research data, including large and complex data types

- gold Open Access which fosters wider collaboration and increased citations

- maximum visibility for your research: over $100 \mathrm{M}$ website views per year

At BMC, research is always in progress.

Learn more biomedcentral.com/submissions 